\title{
Contemporary QSAR Classifiers Compared
}

\section{Supporting Information}

Craig L. Bruce, James L. Melville, Stephen D. Pickett and Jonathan D. Hirst

\section{Parameter tuning SVM for $2.5 D$ descriptor set}

For the polynomial kernel, setting the exponent to 2, with the lower complexity constant, produces the best predictions (see Table S1). Using the RBF kernel, the SVM's performance is more sensitive to the complexity constant. With the default complexity constant, an increased $\gamma$ value gives improved results, which are similar to the default $\gamma$ with a larger complexity constant. Increasing the $\gamma$ with the higher complexity constant improves most datasets further, giving the best result with a RBF kernel.

\begin{tabular}{llllllll}
\hline \multirow{2}{*}{$\begin{array}{l}\text { Complexity } \\
\text { Constant }\end{array}$} & \multicolumn{3}{c}{ Polynomial exponent } & & \multicolumn{3}{c}{ RBF $\gamma$} \\
\cline { 2 - 4 } \cline { 6 - 8 } & 1 & 2 & 3 & & 0.001 & 0.01 & 0.1 \\
\hline 0.05 & 68.7 & $\mathbf{7 7 . 8}$ & 75.5 & & 53.5 & 51.4 & 59.0 \\
1 & 75.7 & 76.1 & 74.0 & & 52.5 & 67.2 & 75.2 \\
50 & 74.1 & 74.0 & 73.6 & & 71.1 & 76.5 & $\mathbf{7 7 . 0}$ \\
\hline
\end{tabular}

Table S1. Mean percentage of correctly classified molecules for different parameters of SVMs on 2.5D descriptor datasets. Values in bold denote the highest accuracy for that kernel. 


\section{Parameter tuning SVM for fragment descriptor set}

The SVM performance, after tuning the complexity constant and one kernelspecific parameter, is shown in Table S2. Altering the complexity constant on the polynomial kernel has little effect on performance, no matter which exponent is used (see Table S2). Using the RBF kernel with the default complexity constant and increasing the $\gamma$ has a positive effect. The higher $\gamma$ setting can give the best results.

\begin{tabular}{|c|c|c|c|c|c|c|}
\hline \multirow{2}{*}{$\begin{array}{l}\text { Complexity } \\
\text { Constant }\end{array}$} & \multicolumn{3}{|c|}{ Polynomial kernel exponent } & \multicolumn{3}{|c|}{ RBF kernel $\gamma$} \\
\hline & 1 & 2 & 3 & 0.001 & 0.01 & 0.1 \\
\hline 0.05 & 74.5 & 73.4 & 73.1 & 51.8 & 53.8 & 59.5 \\
\hline 1 & 75.3 & 73.3 & 73.1 & 62.0 & 72.8 & 76.6 \\
\hline 50 & 73.3 & 73.3 & 73.1 & 75.9 & 74.7 & 73.6 \\
\hline
\end{tabular}

Table S2. Mean percentage of correctly classified molecules for different parameters of SVMs on linear fragment descriptor datasets. Values in bold denote the highest accuracy for that kernel. 